Anglais de spécialité et milieux professionnels

\title{
Proposition d'analyse discursive de trois articles de Paul R. Krugman
}

Séverine Wozniak

\section{OpenEdition}

Édition électronique

URL : http://journals.openedition.org/asp/999

DOI : 10.4000/asp.999

ISBN : 978-2-8218-0396-1

ISSN : 2108-6354

Éditeur

Groupe d'étude et de recherche en anglais de spécialité

Édition imprimée

Date de publication : 1 mars 2004

Pagination : $37-46$

ISSN : 1246-8185

Référence électronique

Séverine Wozniak, « Proposition d'analyse discursive de trois articles de Paul R. Krugman », ASp [En ligne], 43-44 | 2004, mis en ligne le 13 mars 2010, consulté le 23 avril 2019. URL : http:// journals.openedition.org/asp/999; DOI : 10.4000/asp.999

Ce document a été généré automatiquement le 23 avril 2019

Tous droits réservés 


\title{
Proposition d'analyse discursive de trois articles de Paul R. Krugman
}

\author{
Séverine Wozniak
}

\section{Introduction}

1 Les essais et les articles de Paul R. Krugman font partie intégrante des programmes d'économie internationale aujourd'hui. Son manuel, International Economics, écrit conjointement avec Maurice Obstfeld reste une des références en la matière; il a d'ailleurs été réédité en 2002 pour la sixième fois. Krugman est relativement renommé en France ; certains de ses essais ont connu un succès manifeste en librairie, à l'instar de $L a$ mondialisation n'est pas coupable (traduction de Pop Internationalism publiée en 1998). Ancien professeur à Yale, Stanford et au Massachusetts Institute of Technology, Paul R. Krugman enseigne actuellement à Princeton. Chroniqueur régulier du New York Times, il est l'un des économistes américains les plus en vue du moment.

2 Aussi, en tant qu'enseignant, avons-nous à notre disposition un corpus très varié, composé de textes pour spécialistes et de publications non strictement professionnelles. Ses articles font preuve d'une volonté de réelle communication avec le lecteur, par la mise en place d'une stratégie d'écriture caractéristique et l'utilisation d'un réseau de références qui sont présupposées communes à l'auteur et au lecteur.

3 Nous nous proposons d'étudier trois textes intégrés dans nos cours d'anglais pour étudiants LANSAD, plus particulièrement des économistes. Ces textes sont de nature différente et ne relèvent pas tous de la langue de spécialité. Nous commencerons par présenter brièvement ce corpus avant d'y appliquer divers outils de l'analyse pragmatique, lexicographique et stylistique qui se révèlent opératoires. Nous développerons ensuite le résultat de la mise en œuvre, et tenterons de dégager des conclusions quant à la pertinence de l'utilisation de tels documents en cours. 


\section{Présentation du corpus}

\subsection{Pourquoi Paul R. Krugman?}

Dans un article publié à l'occasion de la promotion de Pop Internationalism, Paul R. Krugman (1996b) était présenté comme le plus controversé des économistes américains, non seulement pour la nature de sa réflexion, en particulier sur les réalités du commerce international, mais aussi pour sa façon de critiquer ses confrères et, plus généralement, tous ceux qui se considèrent comme des experts ${ }^{1}$. Ses cibles le définissent comme «un non-conformiste à la critique acerbe ${ }^{2}$ "(Krugman 1996b). Au delà de ses propos, la focalisation sur son style nous parait motivée par le fait qu'il souhaite que le principal trait distinctif de son écriture soit la simplicité ${ }^{3}$. Cet objectif semble atteint puisque le journaliste de Newsweek note qu' «il écrit de façon éloquente et simple pour le public ${ }^{4}$ » (Krugman 1996b).

\section{2. "What Do Undergrads Need to Know about Trade? »}

5 Cet article (dorénavant abrégé WDU) constitue le huitième chapitre de l'ouvrage Pop Internationalism (Krugman 1996a) ${ }^{5}$. Dans cet essai, Paul R. Krugman s'attaque à certains clichés qui apparaissent régulièrement à propos de l'économie internationale. Le cœur de cet extrait est constitué de l'analyse de six opinions erronées sur les conséquences de la mondialisation, que Paul R. Krugman s'applique à identifier pour mieux les combattre. Ce document est donc clairement conçu par l'auteur dans un but didactique (politique également, puisqu'il dénigre ses adversaires). Comme l'indique le titre, il s'agit de rappeler les éléments indispensables à la compréhension des grandes questions de l'économie internationale.

6 Le niveau de langue est neutre, l'article ne comporte pas de termes techniques ni de lexies complexes. Plus formellement, l'auteur est totalement dans son rôle d'enseignant, les destinataires étant l'étudiant, l'érudit inscrit dans un processus d'acquisition d'un savoir portant sur un domaine précis (l'économie internationale), et l'enseignant. Il s'agit $\mathrm{du}$ « premier » Krugman, l'expert, théoricien reconnu du commerce international qui, en tant que chercheur, œuvre à l'avancée de la recherche scientifique dans son domaine et à la divulgation de cette connaissance (vulgarisation). Cet article a été choisi pour être étudié en cours d'anglais car il s'intègre dans la bibliographie des travaux dirigés d'Économie internationale dans notre université.

\section{3. « For Richer » \& « The Tax-Cut Con »}

7 Ces deux articles sont de longs essais ${ }^{6}$ publiés dans le New York Times Magazine (Krugman publie de tels essais deux fois par an, ils sont partiellement traduits pour les lecteurs francophones dans Courrier International ${ }^{7}$ ). Le thème principal de «For Richer " (FR) est la disparition des classes moyennes américaines, du fait de l'accroissement de l'écart de revenu entre les riches et les pauvres. "The Tax-Cut Con " (TCC) est une critique des politiques de réduction d'impôt mises en place par les gouvernements successifs aux États-Unis depuis plusieurs décennies. Le statut de l'auteur est différent de celui de WDU. Krugman est le chroniqueur subjectif de la politique intérieure américaine. En fait, le 
spécialiste et le chroniqueur font appel à deux cadres théoriques distincts : le modèle néo-classique, ou standard, de l'économie internationale et une défense du rôle interventionniste de l'État, d'inspiration keynésienne. Nous avons retenu ces articles pour les étudier en cours de master car ils s'inscrivaient dans l'actualité (venant d'être publiés et commentés sur Internet).

Il convient maintenant de tenter de caractériser ces textes, à travers trois traits : les aspects didactique, ludique et subjectif.

\section{Communiquer le savoir : l'aspect didactique}

Le but affiché de Paul R. Krugman lorsqu'il écrit ces textes est de communiquer un savoir. Les trois textes du corpus seront traités de façon différenciée :

WDU est identifié comme un texte didactique dès les premières lignes :

The problem is that most of what a student is likely to read or hear about international economics is nonsense. What I want to argue in this paper is that the most important thing to teach our undergrads about trade is how to detect that nonsense. (WDU : 117-118)

11 Malgré de nombreuses références aux étudiants ${ }^{8}$, le lectorat du texte est plutôt constitué des collègues de Paul R. Krugman : "We should try to instill in undergrads a visceral negative reaction to statements like this" (WDU : 121). On notera ici l'utilisation du «should» opérateur de modalité, porteur d'une valeur déontique : l'auteur s'inscrit alors en plein dans la relation intersubjective en énonçant une obligation d'après ses propres critères (Bouscaren \& Chuquet 1987 : 55). Il les applique d'ailleurs à lui-même ; il fonde bien sûr le contenu du conseil sur sa propre analyse du commerce international. La stratégie didactique se développe aussi par le recours à des schémas récurrents dans l'argumentation : les six idées erronées (IE) qui sont dénoncées par Krugman dans le texte sont toutes présentées de la même façon : un terme ou une expression entre guillemets ${ }^{9}$, identifiés comme erronés, avec appui sur des éléments factuels et sur :

- une parabole empruntée à un autre auteur (Ingram 1983) (IE $\left.n^{\circ} 1\right)$;

- les idées développées par les maîtres ${ }^{10}$ (IE n²) ;

- une expérimentation pédagogique menée par Krugman (IE $\left.n^{\circ} 3\right)$;

- une anecdote sur l'expérience de chroniqueur de Krugman (IE n³) ;

- une déclaration politique (IE $\left.n^{\circ} 5\right)$;

- des clichés, sous la forme de citations empruntées à d'autres auteurs (IE n², 4 et 6).

Cette juxtaposition de références réalistes participe d'un "effet de réel » qui rend la démonstration plus parlante pour le lecteur. De plus, la structure de l'article elle-même souligne un souci didactique: la problématique et sa justification sont aisément identifiables.

13 En ce qui concerne les deux articles du New York Times Magazine, le lectorat visé est un public d'adultes, la structure n'est donc pas aussi clairement (scolairement) visible, même si les textes sont divisés en sous-parties qui suivent la logique argumentative du discours et se rattachent au style journalistique américain: anecdote (enfance de l'auteur/ description d'un comic strip), données du problème et problématique, perspective historique et comparaisons, conclusion et retour à l'item de départ.

La langue employée par Krugman n'est pas jargonnante, même s'il manie souvent un lexique technique. Le style de WDU est non marqué, comme l'est celui des deux articles, 
avec des passages familiers (dans l'utilisation notamment de formulations orales). Si l'utilisation de la langue technique de spécialistes parait totalement appropriée dans le cas d'un ouvrage pour spécialistes (WDU), nous pouvons peut-être nous interroger sur sa présence dans des articles extraits d'un magazine à visée généraliste. En fait, ces termes nous semblent ici porteurs d'une sorte de marquage socioculturel: les comprendre nécessite la maîtrise de concepts abstraits, et Krugman flatte d'une certaine façon le lecteur, son « Lecteur Modèle » tel que U. Eco (1985) le définit, suggérant l'idée suivante : « ces termes sont techniques et compliqués, je le sais, mais je vous suppose assez instruits pour que cela ne soit pas un problème ». Pour Krugman, la difficulté réside alors dans une assez subtile catégorisation en «mots techniques pour spécialistes » et « mots techniques pour érudits ».

En se posant comme le transmetteur d'un savoir, et par la mise en œuvre de stratégies didactiques variées, Krugman affirme son point de vue comme la vérité. De façon à assouplir justement cet aspect didactique, et de façon à construire une connivence facilitatrice de transmission de savoir - avec le lecteur, il fait appel au ludique.

\section{Bâtir une connivence avec le lecteur : l'aspect ludique}

\subsection{L'ironie}

16 L'aspect ludique apparaît de différentes façons. En premier lieu, par l'ironie, c'est-à-dire la «raillerie particulière, par laquelle on dit le contraire de ce que l'on veut faire entendre » (Le Petit Littré), utilisée constamment par Krugman. Par exemple, il voit en ses cibles des hommes au sens pratique ${ }^{11}$ (WDU : 120), qui, en théorie, ont fait quelques études :

Lester Thurow is a trained economist, who understands comparative advantage. Yet his recent book has been a best-seller largely because it vigorously propounds concepts that unintentionally (one hopes) pander to the clichés of pop internationalism ${ }^{12}$. (WDU : 121) ou :

And here's a radical thought: if the rich get more, that leaves less for everyone else. (FR : 67)

17 Cette ironie apparaît aussi dans l'utilisation de nombreux néologismes lexicaux dans des cas bien précis : là où le néologisme devrait signaler l'introduction d'un nouveau concept (" unique naming of new concepts» Sager 1990: 79), il ne fait que souligner la vacuité intellectuelle des pseudo concepts proposés (selon Paul R. Krugman, qui utilise ici l'argument d'autorité et met clairement en doute la compétence des personnes citées). Le plus évident de ces néologismes est sans doute pop internationalism (dans l'ouvrage éponyme), où la suffixation en -ism connote péjorativement le mot qui, de plus, fait référence à un mouvement artistique (si on veut gloser, l'effet est le suivant : les « Pop Internationalistes » sont des artistes branchés à l'idéologie absconse ${ }^{13}$ ).

-ISM: 'doctrine of', 'practice of as in Calvinism, idealism, impressionism, fanaticism, absenteeism, racism. The items concerning religion, politics, philosophy, and art usually have a corresponding item in -ist to denote adherents and practitioners. (Quirk et al. 1985 : 1549)

La valeur dépréciative de la suffixation en -ism dans le cas d'une implication idéologique - c'est le cas ici - est mise en lumière par Catherine Kerbrat-Orecchioni : 
[...] l'instabilité des investissements axiologiques que l'on observe dans les compétences lexicales tient surtout à la diversité des compétences idéologiques qu'elles reflètent: [...] "poujadisme », « réformisme ", «électoralisme ", " racisme », « sexisme », « jeunisme », etc., [...] fonctionnent régulièrement comme des termes injurieux et peuvent donc être considérés comme marqués en langue [... ]. (Kerbrat-Orecchioni $1980: 76$ )

Ce décalage entre signifiant et signifié est également mis en évidence par les différents tropes présents dans le corpus. Tout d'abord, les comparaisons, où le lien comparé / comparant est explicité par la présence de « like », qui souligne une volonté de clarté :

[...] they illustrated the extent to which corporate leaders now expect to be treated like ancien régime royalty, (FR:64)

In the 1960's, America's great corporations behaved more like socialist republics than like cutthroat capitalist enterprises, and top executives behaved more like public-spirited bureaucrats than like captains of industry, (FR : 66)

We should think of it [the explosion of executive pay] not as a market trend like the rising value of waterfront property, but as something more like the sexual revolution of the 1960's - a relaxation of old strictures, a new permissiveness [...]. (FR: 66)

20 L'étape suivante dans la relation comparé / comparant est la métaphore, où, par l'emploi d'un lien direct, synthétique, le rapport analogique entre signifiant et signifié est une composante de l'aspect subjectif du texte. Ces métaphores sont parfois porteuses d'un double sens et constitutives d'un jeu de mots: "So it's not the invisible hand of the market that leads to those monumental executive incomes; it's the invisible handshake in the boardroom " (FR: 66 - référence à la métaphore d'Adam Smith, qui est rappelée avant d'être détournée), « the rich and tasteless » (FR: 63, référence à un célèbre soap opera), « the man in the gray flannel suit » (FR:64, pour le dirigeant d'entreprise).

\subsection{La dérision et la moquerie}

Ensuite Paul R. Krugman utilise un procédé peut-être moins subtil: la dérision et la moquerie. Le passage précédemment cité à propos de Lester Thurow relève du sarcasme, en particulier au sujet de son best-seller, Head to Head (1992). Notons, puisqu'il peut s'agir pour nous d'un point d'intérêt significatif, que Krugman ne semble pas porter la FASP dans son cœur : dans WDU, il cite Rising Sun, écrit par Michael Crichton et publié en 1992 (adapté pour le cinéma par Philip Kaufman en 1993), comme une source non-négligeable de clichés. L'auteur utilise des guillemets autour de fragments de discours rapportés lorsqu'il veut évoquer ces "clichés du pop internationalism " (WDU : 121). Il montre ainsi qu'il n'assume pas les termes qu'il cite, il s'en distancie pour pouvoir mieux les critiquer ${ }^{14}$. Notons l'aspect totalement rhétorique du procédé : de vraies - ou de fausses ${ }^{15}-$ citations sont données entre guillemets pour être commentées et critiquées par l'auteur. Il s'agit ainsi pour Krugman de parodier, c'est-à-dire d'imiter un style pour rendre les idées ridicules ${ }^{16}$.

What makes parody so amusing often is not simply recognizing what features are

being parodied and why, but also appreciating the parodist's own creative talents:

fusing creativity or wit with critique. (Waugh in Wales 1989 : 338)

Le dispositif de prédilection utilisé par Krugman pour dénoncer les opinions d'après lui erronées est la collocation et les expressions convenues : la concurrence entre nations (« compete in an even tougher world marketplace / be competitive / maintain a standard of living / face an onslaught from new competitors »), le lien emploi - mondialisation (" destroy jobs / 
generate jobs / lose jobs»), le rapport entre le travail effectif des grands présidents directeurs généraux américains et leur paie («provide huge incentives for performance / deliver results »). Il détourne également ces collocations qui deviennent alors ironiques : « Executives who devote their time to creating innovative ways to divert shareholder money into their own pockets probably aren't running the real business very well [...] $)(\mathrm{FR}: 76)$.

\subsection{Le décalage}

Dans les textes étudiés ici, l'humour naît d'un décalage constant entre ce que le lecteur attend et ce qui est donné comme référence. Par exemple, à égalité avec les données statistiques, nous trouvons de petites anecdotes et des histoires personnelles: son expérience d'enseignant et de chroniqueur (WDU), son enfance (FR), ses liens avec les hommes du Président (TCC). Soulignons qu'il s'agit ici d'un travers de l'auteur, sans doute un peu égocentrique et peut-être soucieux de poser les fondations d'un mythe Paul R. Krugman. Il souhaite, à l'instar de Keynes, faire de la science économique un débat de société pour marquer son époque. Par ailleurs, nous sommes en présence de stars de la télévision (Jay Leno, Robert Novak), de quelques présidents directeurs généraux et hommes d'affaires «starisés» (J.P. Morgan, Bill Gates, Jack Welch, Lee Iacocca, Dennis Koslowski, Gerald Levin dont le statut de star est illustré par le renvoi à des magazines tels que Business Week, Forbes ou Fortune). Enfin, les sources sont des articles, mais également des best-sellers d'économie, des romans (Rising Sun de M. Crichton, The Great Gatsby de S. Fitzgerald, The Bonfire of the Vanities de T. Wolfe), et des films. Wall Street d'Oliver Stone est cité et l'une des célèbres répliques du film est même reprise pour illustrer un argument dans l'article « For Richer » :

During the 1980's and 1990's a torrent of academic papers - popularized in business magazines and incorporated into consultants' recommendations - argued that Gordon Gekko was right: greed is good; greed works. (FR : 66)

Remarquons que ces références font partie intégrante du raisonnement, leur rôle est de suggérer une comparaison significative dans l'esprit du lecteur, elles jouent un rôle métaphorique : «The America of «Wall Street» and «The Bonfire of the Vanities» was positively egalitarian compared with the country we live in today" (FR:65). Bien entendu, seules les indications atypiques sont retenues ici; Krugman utilise également des données quantitatives et cite des études (comme celles de Picketty \& Saez et de Goldin \& Margo dans FR, un ouvrage de Galbraith ${ }^{17}$...). Nous pouvons identifier la récurrence d'un déplacement des niveaux de langue : le style utilisé est toujours d'un degré moindre que celui attendu (le style neutre de WDU s'oppose au style académique requis pour The American Economic Review ; pour ce qui est des articles du New York Times Magazine, notons que les occurrences de style oral sont moins abondantes dans TCC que dans FR). Nous pouvons également remarquer l'usage répété des modalités exclamatives ( unfortunately, actually, depressingly »), ce qui accentue encore l'effet de style oral.

La forme ici sert d'appui à la critique, plutôt directe et franche, de l'auteur envers ses confrères principalement. Son rôle est également de bâtir un lien, une connivence avec le lecteur contre un tiers ${ }^{18}$. Krugman fait ainsi un usage régulier de la première personne du pluriel de l'impératif comme stratégie d'intégration auteur / lecteur :

Let's leave actual malfeasance on one side for a moment [...], (FR : 66)

But let's focus on a specific case [...], (FR : 76)

But first, let's set the stage by taking a look at the current state of taxation in

America, (TCC : 56) 
Let's be clear: very few economists think that Clinton's policies were primarily responsible for that miracle, (TCC : 59 )

So let's put a few things back on the table. Let's assume that interest on the public debt will be paid [...]. (TCC : 62)

\section{S'impliquer pour convaincre : l'aspect subjectif}

À travers l'utilisation de diverses techniques rhétoriques, Krugman veut convaincre son lecteur. Il souligne d'ailleurs que l'analyse rhétorique occupe une place non négligeable dans son discours (il étudie « la rhétorique du pop internationalism » WDU : 118).

\subsection{Marqueurs de modalité}

La dimension subjective, c'est-à-dire une prise de position de l'énonciateur vis-à-vis de l'énoncé qui implique un jugement de valeur, est donc constamment présente dans le texte, à travers différents marqueurs récurrents, en particulier ceux de la modalité assertive.

Modality covers all those features of discourse which concern a speaker's or writer's attitude to, or commitment to, the value of applicability of the propositional content of an utterance, and concomitantly, his relationship with whoever he directs the speech act to. (Fowler in Carter \& Simpson 1989 : 95)

Grâce à ce processus, l'énonciateur porte un jugement évaluatif sur son énoncé, il «renforce, nuance ou rectifie la portée de l'assertion qu'il est en train de faire» (Fromilhague \& Sancier 1991 : 88); par l'emploi par exemple du pronom personnel de la première personne du singulier, souvent associé à un verbe porteur d'un point de vue (WDU - 17 occurrences : «I could justify; I think; what I want to argue ; I have found ; I think »; FR - 16 occurrences: "I suspected; I don't mean; I am not exaggerating; I find; I am not suggesting; I could make this argument; I don't think so ; I mean; Am I being too pessimistic? I hope» - qui trouve un écho dans : «my sense; we can be sure ») et de modaux et adverbes assurant des fonctions modales («certainly; probably; definitely; surely; likely; unlikely; possibly»).

\subsection{La connotation}

[...] chaque mot ou lexie peut, auprès de [son] signifié fondamental (dénotation), présenter des valeurs de sens annexes, instables mais souvent prééminentes dans la communication littéraire (connotations). (Perrin-Naffakh 1989 : 24)

Cette dimension subjective apparaît également dans l'usage des marques de connotation : connotation valorisante ou dévalorisante. Nous pouvons alors identifier l'engagement axiologique de l'auteur, qui établit des normes personnelles et juge ensuite d'après cellesci. Ainsi, les idées opposées à sa propre vision des choses sont connotées négativement (définies comme "nonsense, misconceptions, clichés, misrepresentation", elles sont qualifiées de "silly, grossly misleading, phony, distressing, wrong " et " a laughingstock»). En revanche, ses points de vue sont présentés de façon laudative, comme véridiques et définitifs : « the right question, the right answer » (TCC: 62, 59), « realistic quantitative sense » (WDU : 120), « the correct answer » (WDU : 121). 


\subsection{Interpeller le lecteur}

Remarquons enfin le travail sur la présence du lecteur dans le texte :

A more sophisticated pop internationalist like Robert Reich (1991) realizes that the interests of U.S. firms are not the same as those of U.S. workers (you may find it hard to believe that anyone needed to point this out, but among pop internationalists this was viewed as a deep and controversial insight). (WDU : 123)

Ces interpellations, qui participent de l'impression d'oralité, permettent de renforcer le lien auteur / lecteur, d'affirmer et de commenter les propos de l'auteur (elles s'inscrivent dans l'ensemble des dispositifs de l'expression de la subjectivité). Ainsi, elles apparaissent sous forme d'indications de lecture : «Please ignore the numbers for a moment » (WDU : 118), « more on this later » (FR: 64), «But before we get to all that, let's take a look at who gets what » (FR : 64). Pour renforcer cet effet de dialectique auteur / lecteur, Krugman ponctue son discours de "yes", "right» et " of course ». Cet effet apparaît également dans les questions, plus souvent rhétoriques, posées par Krugman :

So we're just talking about shifts within the middle class, right? Wrong: the top 10 percent contains a lot of people whom we would still consider middle class, but they weren't the big winners, (FR : 65)

Is it news that C.E.O.'s [sic] of large American corporations make a lot of money?

Actually, it is. (FR : 64)

Does this mean that the Reagan tax cuts had no effect? Of course not. (TCC : 58)

Ces trois aspects (didactique, ludique et subjectif) se conjuguent donc pour former un style d'écriture tout à fait caractéristique qui révèle peut-être (et notre intention est d'aller ici dans la direction d'une lecture non structuraliste du texte) quelques traits dominants d'une personnalité, ou du moins, de l'image construite d'une personnalité.

\section{Conclusion}

Ces textes ont été proposés à des étudiants de niveau hétérogène. Ils sont habitués - surtout ceux de deuxième cycle - à lire des contributions scientifiques ou des articles de vulgarisation en anglais, qui conjuguent niveau de langue relativement soutenu, et recours à un anglais technique. Ils remarquent tout de suite le contraste, et apprécient de travailler sur la forme autant que sur le fond. Nous l'avons déjà dit, Krugman semble s'appliquer à construire une image à travers son écriture: celle d'une personnalité brillante, au style séduisant, possédant un certain sens du sarcasme et de l'humour. Cependant, il situe volontairement une vision orthodoxe sur le terrain de l'hétérodoxie : il y a vulgarisation du langage mais le référent théorique demeure standard.

Le but de cette étude était de proposer une lecture des textes de Krugman pour convaincre le lecteur de l'intérêt réel de les utiliser dans l'enseignement, car comme il le souligne lui-même, l'économie n'est pas aussi rébarbative qu'on veut bien le dire :

But the honest truth is that what drives me as an economist is that economics is fun 19. I think I understand why so many people think that economics is a boring subject, but they are wrong. On the contrary, there is hardly anything I know that is as exciting as finding that the great events that move history, the forces that determine the destiny of empires and the fate of kings, can sometimes be explained, predicted, or even controlled by a few symbols on a printed page. We all want power, we all want success, but the ultimate reward is the simple joy of understanding. (Krugman 1995 : site) 
L'auteure remercie les chercheurs du Laboratoire d'Économie de la production et de l'intégration internationale - Économie politique de l'intégration internationale et du développement qui ont commenté une version antérieure du texte. Elle reste cependant seule responsable des éventuelles erreurs et imprécisions.

\section{BIBLIOGRAPHIE}

\section{Références du corpus}

Articles de Paul R. Krugman

1996a. « What Do Undergrads Need to Know about Trade? », In Pop Internationalism. Cambridge :

MIT Press, 117-125. [WDU]

1996b. « The Great Debunker ». Newsweek, 4 mars.

2002. « For Richer ». New York Times Magazine, 20 octobre, 62-67, 76-77, 141-142. [FR]

2003. « The Tax-Cut Con ». New York Times Magazine, 14 septembre, 54-62. [TCC]

Sites web

Site officiel http://www.wws.princeton.edu/ pkrugman/ (« How I work » 1993 ; « Incidents from my career » 1995).

« The Unofficial Paul Krugman Archive » http://www.pkarchive.org/.

Références bibliographiques

Bouscaren, Janine \& Jean Chuquet. 1987. Grammaire et textes anglais, Guide pour l'analyse linguistique. Paris : Ophrys.

Carter, Ronald \& Paul Simpson (dir.). 1989. Language Discourse and Literature : An Introductory Reader in Discourse Stylistics. Londres : Unwin Hyman.

Cuddon, J. A. 1991. The Penguin Dictionary of Literary Terms and Literary Theory. Londres : Penguin Books.

Eco, Umberto. 1985. Lector in Fabula. Paris : Grasset \& Fasquelle.

Fromilhague, Catherine \& Anne Sancier. 1991. Introduction à l'analyse stylistique. Paris : Bordas. Ingram, James. 1983. International Economics. New York : Wiley.

Kerbrat-Orecchioni, Catherine. 1980. L'Énonciation : de la subjectivité dans le langage. Paris : Armand Colin.

Krugman, Paul R. \& Maurice Obstfeld. 2002. International Economics. Boston : Addison Wesley.

Perrin-Naffakh, Anne-Marie. 1989. Stylistique Pratique du commentaire. Paris : Presses

Universitaires de France.

Quirk, Randolf, Sidney Greenbaum, Geoffrey Leech \& Jan Svartvik. 1985. A Comprehensive Grammar of the English Language. Londres \& New York : Longman. 
Sager, Juan C. 1990. A Practical Course in Terminology Processing. Philadelphie \& Amsterdam : John Benjamins Publishing Company.

Sokal, Alan \& Jean Bricmont. 1997. Impostures intellectuelles. Paris : Odile Jacob.

Truss, Lynne. 2003. Eats, Shoots and Leaves. Londres : Profile Books.

Wales, Katie. 1989. A Dictionary of Stylistics. Londres \& New York : Longman.

\section{NOTES}

1. "What most students need to be prepared for, however, is a world in which TV "experts", best-selling authors, and \$30,000-a-day consultants do not understand budget constraints, let alone comparative advantage ». (WDU : 124)

2. «A sharp-tongued maverick».

3. L'en-tête d'un chapitre de l'article «How I work», publié en 1993, où Paul R. Krugman développe sa méthodologie de recherche, est «Simplify, simplify». Dans Pop Internationalism, Krugman déclare: "What I eventually realized was that an effective answer to pop internationalism would require a new kind of writing. I would have to write essays for non-economists that were clear, effective, and even entertaining - otherwise, nobody would read them » (p.xi). L'intérêt que porte Krugman au style et à l'écriture pourrait constituer un axe de recherche intéressant. Il commence la préface de The Age of Diminished Expectations.U.S. Economic Policy in the 1990s (1994), par exemple, par un développement sur les différents modes et niveaux de langue dans les écrits économiques (scientifique, journalistique et "d'aéroport ", qui définit le style des best-sellers économiques, dont il critique régulièrement les auteurs).

4. «[...] he writes eloquently and simply for the public».

5. Le texte avait déjà été publié dans The American Economic Review en mai 1993.

6. FR : 8041 mots, TCC : 7145 mots

7. FR : « Main basse sur l'Amérique » (09/01/03), TCC : «Baisse des impôts : le Grand Bluff, Qui veut la peau de l'État providence?» (25/09/03).

8. De façon caractéristique, le terme «student/s » apparaît quinze fois dans le texte, « undergrads " apparaît dans le titre et dans le texte (six fois) - notons l'utilisation d'un mot appartenant à un registre de langue plus familier. "Teach » apparaît huit fois, "drive home the point » est utilisé deux fois.

9. Observons la même utilisation des guillemets pour « the death tax » (FR et TCC) et «starving the beast » (TCC).

10. Ricardo, évoqué deux fois.

11. «Practical men ».

12. ou encore: "People who say things like this believe themselves to be smart, sophisticated, and forward-looking " (WDU : 118), «[...] what passes for educated discussion about international trade » (WDU : 118), « charlatans and cranks » (TCC : 58, à propos des tenants de l'économie de l'offre).

13. Cette technique est réutilisée à l'envi : «starve-the-beasters » et «supply-siders » (TCC).

14. Comme Lynne Truss l'écrit de façon très imagée : «Inverted commas (speech marks, or quotes) are sometimes used by fastidious writers as a kind of linguistic rubber glove, distancing them from vulgar words or clichés they are too refined to use in the normal way » (2003:148).

15. "OK, I confess: it's not a real quotation. I made it up as a sort of compendium of popular misconceptions about international trade. But it certainly sounds like the sort of thing one reads or hears all the time $[. .]$.$» . (WDU : 118)$

16. Rappelons que la parodie comme instrument de la critique fondamentale et virulente avait le vent en poupe à l'époque où le texte a été repris dans Pop Internationalism. Le célèbre canular de 
A. Sokal et J. Bricmont a en effet été publié dans le numéro de printemps / été 1996 de Social Text et, immédiatement dévoilé par Sokal, il a déclenché une tempête de réactions chez les intellectuels américains.

17. The New Industrial State.

18. "The rich have always been different from you and me. » (FR_: 64)

19. Nous sommes alors à l'opposé de « the dismal science ».

\section{RÉSUMÉS}

Paul R. Krugman est un des spécialistes éminents de l'économie internationale aujourd'hui. Les textes étudiés ici traitent des points fondamentaux du commerce international ( What Do Undergrads Need to Know about Trade? »), des différences de revenu aux États-Unis («For Richer »), et des politiques de réduction d'impôt de l'administration américaine ("The Tax-Cut Con»). À travers cette analyse, nous tentons de montrer que Krugman fonde sa stratégie discursive sur trois pôles distincts : le didactique, le ludique et le subjectif. Le but premier de Krugman est d'expliquer; aussi, convient-t-il d'identifier clairement ses méthodes d'argumentation, son lectorat et de catégoriser la langue employée. L'aspect ludique apparaît à travers la présence récurrente dans le corpus de l'ironie, la dérision et la moquerie, et un décalage constant entre ce que le lecteur attend et ce qui lui est proposé. Enfin, la subjectivité s'exprime par le jeu avec la modalité, la connotation et les stratégies de renforcement du lien auteur/lecteur.

Paul R. Krugman is one of the most prominent specialists of international economics today. The texts studied here deal with the fundamentals of international trade ("What Do Undergrads Need to Know about Trade?"), the income gap in the U.S. ("For Richer"), and the tax cut policies implemented by the American administration ("The Tax-Cut Con"). In this analysis, we will try to show that Krugman's discourse strategy follows three main axes: didactic, playful and subjective. Krugman's main purpose is to explain, therefore, we must clearly identify his methods of argument, his readership and characterize his language. Playfulness is shown in the recurrence of irony, derision and mockery, and a constant discrepancy between what the reader expects and what s/he is given to read. Finally, subjectivity is expressed through the play on modality, connotation and the strategies used to strengthen the bond between the writer and the reader.

\section{INDEX}

Keywords : discourse analysis, economic English, Krugman (Paul R.), new theory of international trade, newspaper article

Mots-clés : analyse du discours, anglais économique, article de presse, Krugman (Paul R.), nouvelle théorie de l'économie internationale

\section{AUTEUR}

\section{SÉVERINE WOZNIAK}

